Linguistic Synaesthesia in Turkish: A Corpus-Based Study of Cross-Modal Directionality

\author{
Alper Kumcu ${ }^{1}$ \\ ${ }^{1}$ Department of Translation and Interpreting, Hacettepe University
}

Author Note

THE REVISED AND PEER-REVIEWED VERSION OF THIS WORK HAS BEEN PUBLISHED IN METAPHOR AND SYMBOL ON 03 OCTOBER 2021:

https://www.tandfonline.com/doi/full/10.1080/10926488.2021.1921557.

PLEASE DO NOT CITE OR COPY THIS PREPRINT VERSION.

We have no conflicts of interest to disclose. Correspondence concerning this article should be addressed to Alper Kumcu, Hacettepe Üniversitesi, Edebiyat Fakültesi, Mütercim-Tercümanlık Bölümü, Çankaya/Ankara, 06800 Turkey. Email: alperkumcu@hacettepe.edu.tr ORCID: https://orcid.org/0000$\underline{0003-0844-3562}$ 
Word count: 247 (abstract)

Word count: 7824 (manuscript including abstract) 


\begin{abstract}
Linguistic synaesthesia (i.e., synaesthetic metaphor, intrafield metaphor or cross-modal metaphor) refers to instances in which expressions in different sensory modalities are combined as in the case of sweet (taste) melody (sound). Ullmann (1957) and later, Williams (1976) were first to show that synaesthetic transfers seem to follow a potentially universal pattern that goes from the lower (i.e., touch, taste and smell) to higher senses (i.e., hearing and sight) but not the other way around (e.g., melodious sweetness) Studies across languages, cultures, domains, and text types presented mixed results as to the universality claim of cross-modal mappings in linguistic synaesthesia (e.g., Jo, 2019; Strik Lievers, 2015; Zhao et al., 2019). To extend results to an underrepresented language and thus, to test the universality of the directionality principle, 5699 cases of linguistic synaesthesia in written and spoken Turkish were investigated using a general-purpose, large corpus. Results show that except for the transfers from smell to hearing which is unidirectional, synaesthetic transfers in Turkish do not comply with the directionality principle in the strictest sense. Although most transfers that follow the canonical direction were also significantly more frequent, there were instances of "backward transfers". Further, two of the backward transfers (i.e., from smell to touch and from taste to touch) were significantly more frequent than their canonical counterparts (i.e., from touch to smell and from touch to taste). Results are compared against synaesthesia in other languages and discussed in the framework of linguistic universals and embodied cognition. Supplemental materials: https://osf.io/2unvy
\end{abstract} Keywords: linguistic synaesthesia, synaesthetic metaphor, transfer directionality, perceptual modality, sensory linguistics, Turkish 


\section{Linguistic Synaesthesia in Turkish: A Corpus-Based Study of Cross-Modal Directionality}

Synaesthesia is a rare psychological condition of cross-modal sensory and conceptual mapping in which stimulation of one sensory modality (e.g., hearing) leads to an involuntary stimulation in another, unrelated modality (e.g., sight) (Ramachandran \& Hubbard, 2001). For instance, synaesthetic individuals might see a particular colour or a pattern whenever they hear a particular sound (e.g., D-flat is brown). Such cross-modal experiences can emerge between touch, smell, taste or even between modalities beyond Aristotelian five-sense system such as lexical - gustatory synaesthesia, that is, associations between words and tastes (Ward \& Simner, 2003).

In contrast to psychological synaesthesia, linguistic synaesthesia is not restricted to a small population, rather extends to different languages and cultures as discussed below. This linguistic phenomenon mostly occurs within adjective-noun and adverb-verb constructions where a perceptual head and a perceptual modifier are combined as in the case of sweet melody or to smell sweet. Linguistic synaesthesia is typically understood and studied in the context of metaphors (see Shen \& Aisenman, 2008). ${ }^{1}$ Metaphors, in essence, provide an avenue to "understand and experience one kind of thing in terms of another" and are a fundamental element of human thought rather than being just a "matter of words" (Lakoff \& Johnson, 1980a). Conceptual metaphors connect two distinct domains referred to as source and target as illustrated in the following example (Lakoff \& Johnson, 1980b):

(1) His brain is packed with interesting ideas.

The metaphorical expression in the sentence above follows the metaphorical concept of "THE MIND IS A CONTAINER". The concept in the source domain (i.e., container) is mapped onto the concept in the target domain (i.e., mind). Thus, container, a relatively more concrete concept, allows for

\footnotetext{
${ }^{1}$ That said, whether linguistic synaesthesia is a metaphor is debated (see Winter, 2019).
} 
reasoning about the mind. Synaesthetic metaphors display a similar pattern yet source and target concepts belong to different sensory modalities. Consider the following examples:

(2) A warm colour in the bedroom like yellow will match your furniture.

(3) This is the place where she has heard the sweet melody for the first time.

In the case of warm colour, the adjective warm in the source domain of one sensory modality (i.e., touch) is mapped onto the noun colour in the target domain of another sensory modality (i.e., sight). In other words, warm colour is a case of touch - sight transfer and similarly, sweet melody is a case of taste - hearing transfer. ${ }^{2}$ As in conventional metaphors, linguistic synaesthesia allows for understanding the target perception (e.g., sight in the case of warm colour) in terms of the source perception (e.g., touch in the case of warm colour).

A central question in linguistic synaesthesia is the directionality between senses: Does the mapping between source and target modalities follow a certain principle or can any sensory modality be mapped onto any other? Upon investigating over 2000 synaesthetic metaphors in the $19^{\text {th }}$ century poetic texts in English, French and Hungarian; Ullmann (1957) developed a hierarchical linear model of linguistic synaesthesia which shows the transfer directionality between senses, known as the directionality principle (see also Tsur, 1992). According to the directionality principle, synaesthetic transfers are not random but follow a certain pattern from "lower" (and proximal) perceptual modalities (i.e., touch, taste and smell) to "higher" (and distal) ones (i.e., hearing and sight) (see Figure 1). The principle rests on the presumption that there is a universal hierarchy of the senses, with sight as the most distinct, that is, differentiated and touch as the least distinct sense (see Majid et al., 2018). Along

\footnotetext{
${ }^{2}$ Some authors make a distinction between strong and weak synaesthesia (e.g., Werning et al., 2001). Accordingly, warm colour is a case of strong synaesthesia as both domains are perceptual and cold heart is a case of weak synaesthesia as only source domain is perceptual. Linguistic synaesthesia in the current study is restricted to strong synaesthesia as per this distinction.
} 
with that, frequencies in Ullmann (1957) indicated that touch is the predominant source but hearing, rather than sight, is the predominant target for synaesthetic transfers.

\section{Figure 1}

A Simplified Version of Ullmann's Model (1957)

\section{Touch $>$ Taste $>$ Smell $>$ Hearing $>$ Sight}

It is important to note that such a hierarchy between senses dictates a unidirectional mapping.

That is, the only possible direction is from left (lower senses) to right (higher senses) in the model.

Accordingly, transfers in warm colour (from touch to sight) and sweet melody (from taste to hearing), for example, are in agreement with the principle. However, hypothetical examples such as coloured warmness (from sight to touch) or melodious sweetness (from hearing to taste) are not expected to occur due to the inverse mapping between the source and target perceptual domains. Williams (1976) proposed a slightly revised version of this model based on data drawn from everyday English and Japanese, in which sight is grouped into two categories as dimension and colour (see Figure 2).

\section{Figure 2}

Williams' Model of Synaesthetic Transfers (1976)

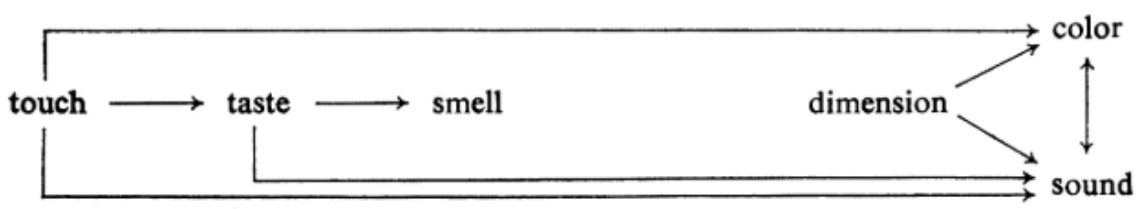

Although transfer routes in Ullmann's model and Williams' model are relatively similar, Williams (1976) further suggested that the directionality principle might be universal and thus, can be generalised 
to linguistic synaesthesia across languages, cultures and historical periods. Indeed, the transfer pattern was found in poetic Hungarian (Erzsébet, 1974), literary American English and German (Day, 1996), everyday French (Wise, 1997), poetic Hebrew (Shen, 1997; Shen \& Cohen, 1998), everyday Indonesian (Shen \& Gil, 2008), literary and non-literary Chinese (Yu, 1992, 2003) in addition to poetic English, French and Hungarian and non-poetic English and Japanese as discussed above. ${ }^{3}$ It was argued that the selective and potentially universal pattern might be based on a biological foundation that is hardwired to the brain and thus, transcends linguistic borders (Marks, 1996; Rakova, 2003; Ronga et al., 2012). Further, Shen and Aisenman (2008) reported that the conventional higher-to-lower structure is better remembered and is judged as easier to build a context for than the inverse mapping. They also argued that linguistic synaesthesia follows the same rules in conventional metaphorical transfers and mapping go from somehow more concrete senses (i.e., touch, taste, smell) to less concrete ones (i.e., hearing and sight) (see also San Roque et al., 2015).

However, recent studies on various languages such as English (Strik Lievers, 2015), Italian (Strik Lievers, 2015), Mandarin Chinese (Zhao et al., 2018) and Korean (Jo, 2019) have challenged the universality claim of the directionality principle and suggested that cross-modal transfers may not be "strictly" unidirectional. As one example, Strik Lievers (2015) investigated about 500 cases of synaesthesia in English and Italian drawn from the ukWaC and itWaC corpora (Baroni et al., 2009) respectively. Results indicated that although most of the transfers occur in the expected, that is, lowerto-higher direction, there are instances of backward transfers such as bitter cold (from taste to touch). It was, therefore, suggested that the principle of mapping directionality is to be interpreted as a tendency

\footnotetext{
${ }^{3}$ See also Evans and Wilkins (2000) and Viberg (1983) for a similar universality in the extension of perception verbs to describe other sensory modalities and Strik Lievers and Felice (2019) for semantic extension in sensory adjectives.
} 
of frequency rather than an exclusively unidirectional and universal constraint on linguistic synaesthesia. Along with that, touch emerged as the most frequent source, while hearing served as the most frequent target both in English and Italian, supporting Ullmann (1957).

Zhao et al. (2019) used a similar corpus-based approach and identified 8082 instances of synaesthesia in the Sinica Corpus (Chen et al., 1996). Results revealed that synaesthesia in Mandarin Chinese does not strictly and merely follow the conventional mapping pattern either but rather, display three types of tendencies: (1) Unidirectional or rule-based mapping which proceeds only in one direction between two senses but not in the reverse direction (from touch to hearing, from touch to smell and from taste to hearing in the study). (2) Frequency-based mapping as demonstrated in English and Italian (Strik Lievers, 2015) which shows a dominant tendency. For instance, the mappings from touch to sight had a higher frequency than the mappings from sight to touch in the study. (3) Bidirectional mapping that shows no clear preference and goes both ways almost equally (only between touch and taste in the study). Touch was found as the most frequent source domain as previously shown in Indo-European languages.

Lastly, Jo (2019) studied 100 instances of synaesthetic instances in Korean obtained from the Korean National Corpus (Kang \& Kim, 2004). It was demonstrated that the mapping direction, on the whole, is congruent with the Ullmann's hierarchical distribution. However, synaesthesia directionality was not unidirectional yet frequency-based as there were inverse transfer types (i.e., from sight to touch, from sight to smell and from hearing to sight) extending previous findings (Strik Lievers, 2015; Zhao et al., 2019). In line with the data from other languages, results showed that the predominant sensory source was touch and the predominant sensory target was hearing.

Taken together, studies illustrated above suggest that there is abundant evidence from several languages and domains that verifies the universality of the directionality principle. Further, touch and hearing seem to occupy source and target domains consistently across languages. However, attested 
transfers in recent studies indicate that the directionality in linguistic synaesthesia is more complex than previously shown and cannot be classified as rigidly unidirectional. Against this background, the current study investigates the directionality of linguistic synaesthesia in Turkish. Specifically, three research questions related to Turkish linguistic synaesthesia were examined:

(1) Do synaesthetic instances show a specific transfer pattern or do source and target domains map onto each other randomly?

(2) Is the transfer pattern, if any, rule-based (unidirectional) or frequency-based? In other words, are backward transfers possible?

(3) What are the most frequent sensory source and sensory target domains?

The majority of the previous results were obtained from the languages within Indo-European language family (i.e., English, German, French and Italian) along with other language families (Uralic, Austronesian, Japonic, Sino-Tibetian and Koreanic). In this respect, the current study is important as it presents informative data on synaesthesia from an untested language within an untested language family. Whether and/or to what extent Turkish linguistic synaesthesia adheres to the directionality principle could provide support for either camp of the directionality debate.

\section{Method}

The present study reports a corpus-based study according to the distinction introduced by Tognini-Bonelli (2001). To be more precise, the research questions described above were tested based on the analyses of the data extracted from the Turkish National Corpus (TNC) which can be accessed at https://www.tnc.org.tr (TNC Team, 2018). Linguistic synaesthesia was identified and extracted with a semi-automatic metaphor extraction procedure developed in Strik Lievers et al. (2013) as described below. Data were analysed and visualised in the R programming language and environment ( $\mathrm{R}$ Core Team, 2020).

\section{Corpus}


TNC is a well-established, balanced and representative general-purpose corpus of contemporary Turkish. It involves 50 million word tokens and it consists of samples of textual data across a wide variety of genres covering 20 years (1990-2009). The written component consists of texts produced in both literary and non-literary domains, including poetry, short stories, and journalistic articles on various topics. Transcriptions from spoken data constitute $2 \%$ of the TNC's database, which involves spontaneous, everyday conversations and speeches collected in particular communicative settings such as meetings and lectures (Aksan et al., 2012).

Therefore, data extracted from the TNC cannot be described as poetic or domain-specific. Thus, instances of synaesthesia can be said to represent language rather than a specific work of art, domain or genre. Further, the TNC allows for a sufficient number of occurrences due to its large volume. In sum, the TNC can be said to satisfy the requirements of a corpus to conduct a comprehensive and finegrained investigation on linguistic synaesthesia and to test the validity of transfer hierarchy in Turkish (see Strik Lievers, 2015).

\section{Data Retrieval}

The semi-automatic metaphor extraction procedure followed in the current study is composed of three steps as (1) compilation of perceptual lexemes, (2) extraction and annotation, and (3) manual inspection:

(1) Compilation of perceptual lexemes. The first and the most crucial step of the extraction procedure is compiling an inventory of perception-related lexemes. Three practical decisions were to be made at this stage to define the scope of the data retrieval:

(a) What makes a lexeme perceptual? To overcome the problem of determining whether a lexeme is related to perception, we followed the conventions in the literature. Accordingly, the list of perceptual lexemes in the current study was compiled by taking 425 lexemes in English in Strik Lievers (2015) and 199 lexemes in Chinese in Zhao et al. (2018) as example. Perceptual adjectives and nouns 
that are compatible with Turkish were translated while less frequent and/or unfamiliar lexemes were omitted. The list was then expanded with synonyms, antonyms and hyponyms using two electronic Turkish thesauri: Dokuz Eylül University Turkish Thesaurus (http://nlpapps.cs.deu.edu.tr/esveyakin) and Mythes-Tr Turkish Thesaurus (https://github.com/maidis/mythes-tr).

(b) Which word classes are to be referred to classify perceptual lexemes? Both Strik Lievers (2015) and Zhao et al. (2018) have indicated that linguistic synaesthesia is found primarily (but not exclusively) in adjective-noun combinations. Thus, the list in the present study included perceptual adjectives and perceptual nouns. Although verbs were not included per se, several verbal nouns such as parıldama - sparkling (sight) or konuşma - talk/ing (hearing) were evaluated as verbs as well depending on the cases in which adjectives functioned as adverbs. Further, wildcards were used for certain lexical items such as * sesi-sound of * or * kokulu - * scented to access more instances related with perceptual lexemes.

(c) Which senses are to be referred to classify perceptual lexemes? Classification of human senses has been a highly controversial issue and there is no consensus on the number of senses (e.g., Gray, 2005; Keeley, 2002; Macpherson, 2011; Merleau-Ponty, 1960). Beyond the Aristotelian system involving touch, taste, smell, hearing and sight, relatively unorthodox views identify as many as 33 senses varying from interoception (e.g., thirst, hunger, blood pressure etc.) to mechanoreception (balance, rotation, acceleration etc.) (e.g., Craig, 2002). While future studies are recommended to explore linguistic synaesthesia in understudied senses, the scope of the current study is limited to the traditional five-senses model for a well-defined criterion of synaesthesia in line with the conventions in the literature and thus, to attain comparable results. Perceptual adjectives and nouns were assigned to the five senses based on the evaluations of 12 native Turkish speakers. Even with external evaluation, tagging perceptual lexemes with senses is a challenging task because perceptual experience cannot be limited into one sense easily and more often than not, perceptual lexemes spread over multiple senses 
in the case of "multisensory adjectives" (see Lynott \& Connell, 2009; Winter, 2019). Multisensory adjectives in the list such as $a c l$, which can be translated into English as both painful (touch) and spicy (taste), were not excluded and were assigned to a single sense according to the frequency of the preferred sense in the evaluations for the ease of data presentation and analysis. ${ }^{4}$ However, each instance with a multisensory adjective was interpreted in its context as detailed below. The final list consisted of 697 perception-related lexemes distributed into five senses (see Table 1). Comparing the lexemes in the current study against those in Zhao et al. (2018) revealed a similar distribution: In both studies, sight was the sensory domain with the most lexicalised adjective followed by touch. The list of perceptual lexemes used in the present study can be accessed at https://osf.io/ycj29.

\section{Table 1}

The Distribution of the Perceptual Lexemes in Raw Frequencies and Percentages (in Parentheses)

\begin{tabular}{lrrr}
\hline Sense & Adjective & Noun & $\Sigma$ \\
\hline Sight & $135(19.37)$ & $82(11.76)$ & $217(31.13)$ \\
Hearing & $31(4.45)$ & $277(39.74)$ & $308(44.19)$ \\
Touch & $51(7.32)$ & $41(5.88)$ & $92(13.20)$ \\
Taste & $33(4.73)$ & $24(3.44)$ & $57(8.18)$ \\
Smell & $8(1.15)$ & $15(2.15)$ & $23(3.30)$ \\
$\Sigma$ & $258(37.02)$ & $439(62.98)$ & $697(100)$ \\
\hline
\end{tabular}

${ }^{4}$ Only one lexeme, ifade, was assigned to both sight (as in look, expression) and hearing (as in declaration) as both meanings received the same number of preferences. 
(2) Extraction and annotation. TNC was searched for all instances of pre-defined perceptual adjectives and their spelling variants. Concordance lines displayed in a $-5,5$ window span (i.e., 5 lexical items before and after the queried lexeme) were scrutinised to distinguish synaesthetic instances. According to our definition discussed above, a synaesthetic instance involves a perceptual head (a noun or a verb) and a perceptual modifier (an adjective or an adverb) from the lexeme list belonging to different sensory modalities such as tat/ı şarkı - sweet song (from taste to hearing) or tatlı tatı konuşmak - to talk sweetly (from taste to hearing). ${ }^{5}$

Source and target domains in synaesthetic occurrences were then annotated with one of the five senses. The source of the mapping is represented by the adjective or the adverb and the target of the mapping is represented by the noun or the verb. To illustrate, tat/ı - sweet (taste) is the source of the mapping and şarkı-song (hearing) is the target of the mapping in tatlı şarkı-sweet song.

(3) Manual inspection. Adopting a corpus-based approach allowed us to access and examine a wide range of synaesthetic instances in a large collection of literary and non-literary texts and transcriptions of natural speeches efficiently. That said, a semi-automatic extraction method may easily result in false-positive instances (see Gibbs, 2010; Hunston, 2002). Accordingly, occurrences extracted from the concordance lines were manually inspected with close reading and were then interpreted as synaesthesia or not. If the context given with a $-5,5$ window span fell short to decide, the full sentence was inspected. If the full sentence was not sufficient for verification either, the occurrence was excluded altogether.

Instances used metaphorically and/or figuratively themselves were included since the literal sources of these metaphorical uses are originally perceptual. For example, sıkı şarkı-solid song, which

\footnotetext{
${ }^{5}$ Some instances were distributed over the sentence such as ...damağımda çınlayan o büyülü tadıyla... ... with the magical taste (taste/target) that echoes (hearing/source) on my palate...
} 
means a song of superior quality, was considered as synaesthesia in which the source in touch domain (i.e., sıkı-solid) is mapped onto the target in hearing domain (şarkı-song).

Synaesthetic instances with multisensory adjectives were manually inspected and interpreted case by case: An occurrence was considered as synaesthesia if the context suggested that the lexeme belongs to one specific sensory domain. However, occurrences with ambiguous contexts which suggested multiple sensory domains were excluded. Consider pürüzsüz - smooth as an example. Pürüzsüz was classified under touch in the lexeme list. That said, instances with pürüzsüz yüz - smooth face and similar cases from touch to sight such as pürüzsüz görünüm - smooth appearance and pürüzsüz renk - smooth colour were excluded from the synaesthesia sample since smoothness of a face, an appearance or a colour can be perceived by seeing as well as touching. On the other hand, instances with pürüzsüz ses - smooth voice were included in the sample as long as the context allowed since "smoothness" of a voice refers to the experience of touching rather than the experience of seeing. Despite all the effort, it is important to acknowledge that the degree of metaphoricity and the strength of synaesthesia between instances in the sample vary. As the last step, extracted data were cleaned for the exact duplicates.

\section{Results}

\section{Descriptive Analysis}

157,440 concordance lines accessed through 258 adjectives in five perceptual modalities were inspected. As a result, 5699 instances of linguistic synaesthesia were extracted. Turkish Linguistic Synaesthesia Corpus (TLSC) as a sub-corpus of the TNC including 60,138 word tokens was then formed with 5699 synaesthetic instances. Raw frequencies of the attested transfers and their percentages in TLSC were given in Table 2. TLSC with all occurrences can be accessed at https://osf.io/v82cs.

\section{Table 2}


Raw Frequencies and Percentages (in Parentheses) of Synaesthetic Transfers in TLSC

\begin{tabular}{|c|c|c|c|c|c|c|c|}
\hline & \multicolumn{7}{|c|}{ Target } \\
\hline & & Sight & Hearing & Touch & Taste & Smell & $\Sigma$ \\
\hline \multirow{6}{*}{ 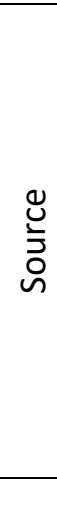 } & Sight & & $1496(26.25)$ & $151(2.65)$ & $7(0.12)$ & $35(0.61)$ & 29.63 \\
\hline & Hearing & $98(1.72)$ & & $5(0.09)$ & $4(0.07)$ & $x$ & 1.88 \\
\hline & Touch & 1056 (18.53) & 1168 (20.49) & & $44(0.77)$ & 224 (3.93) & 43.72 \\
\hline & Taste & 495 (8.69) & $659(11.56)$ & 94 (1.65) & & 118 (2.07) & 23.97 \\
\hline & Smell & $10(0.18)$ & $17(0.30)$ & $17(0.30)$ & $1(0.02)$ & & 0.80 \\
\hline & $\Sigma$ & 29.12 & 58.60 & 4.69 & 0.98 & 6.61 & 100 \\
\hline
\end{tabular}

A general inspection of the raw frequencies indicates that there are 19 transfer types between sensory modalities in Turkish rather than 20 possible transfer types among any two of five senses. The only unavailable transfer type was from hearing to smell. Results showed that touch is the most frequent source (43.72\%) and hearing is the most frequent target (58.60\%) (see Figure 3 and Figure 4). Chi-square tests demonstrated that there are significant differences in the frequency of instances between all senses as source and target domains [hearing: $x^{2}(1)=2997.9, p<.0001$; touch: $x^{2}(1)=$ 1777.2, $p<.0001 ;$ taste: $x^{2}(1)=1199.6, p<.0001 ;$ smell: $x^{2}(1)=259.27, p<.0001$ ] except for sight; $x^{2}(1)$ $=0.25, p=.62$.

\section{Figure 3}

Chord Diagram Showing the Frequency of the Five Senses as the Source and Target Domains 


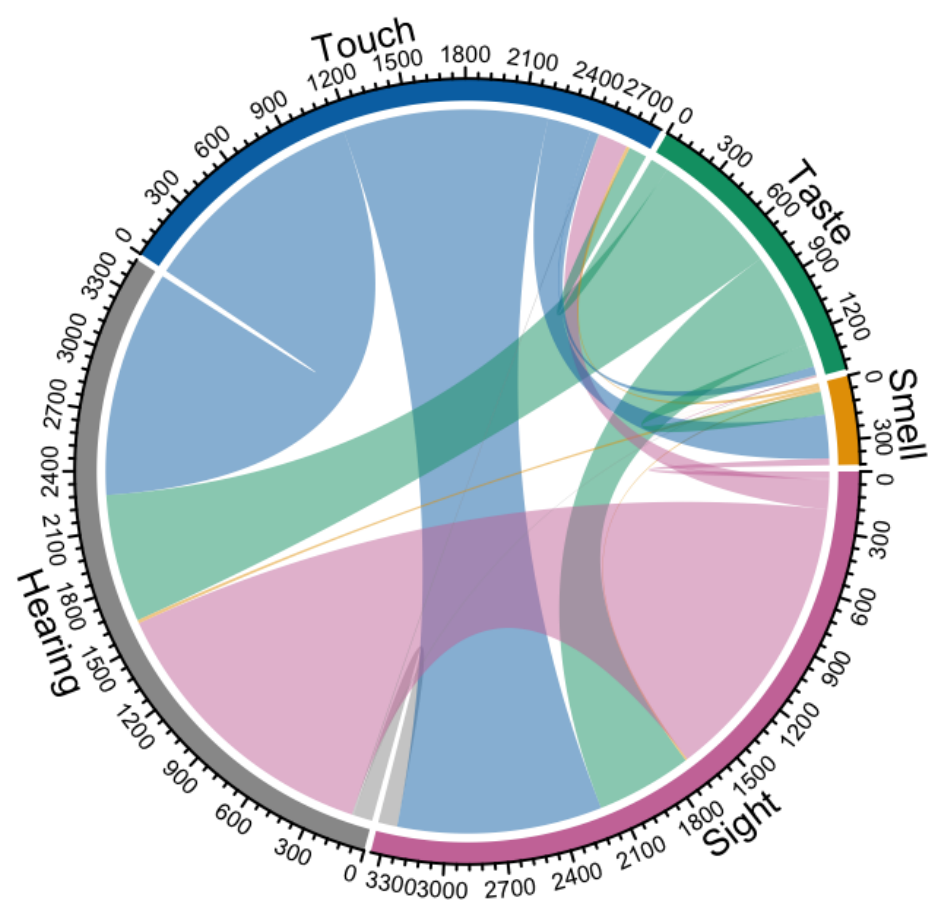

Note. Each sense is represented by the circle segments and frequency is represented by the width of the flow at the beginning and endpoints. Tick marks on the circle segments show the number of instances.

\section{Figure 4}

Horizontal Bar Plots Showing the Frequency of Synaesthetic Occurrences across Senses Broken Down by the Source and Target Domains 

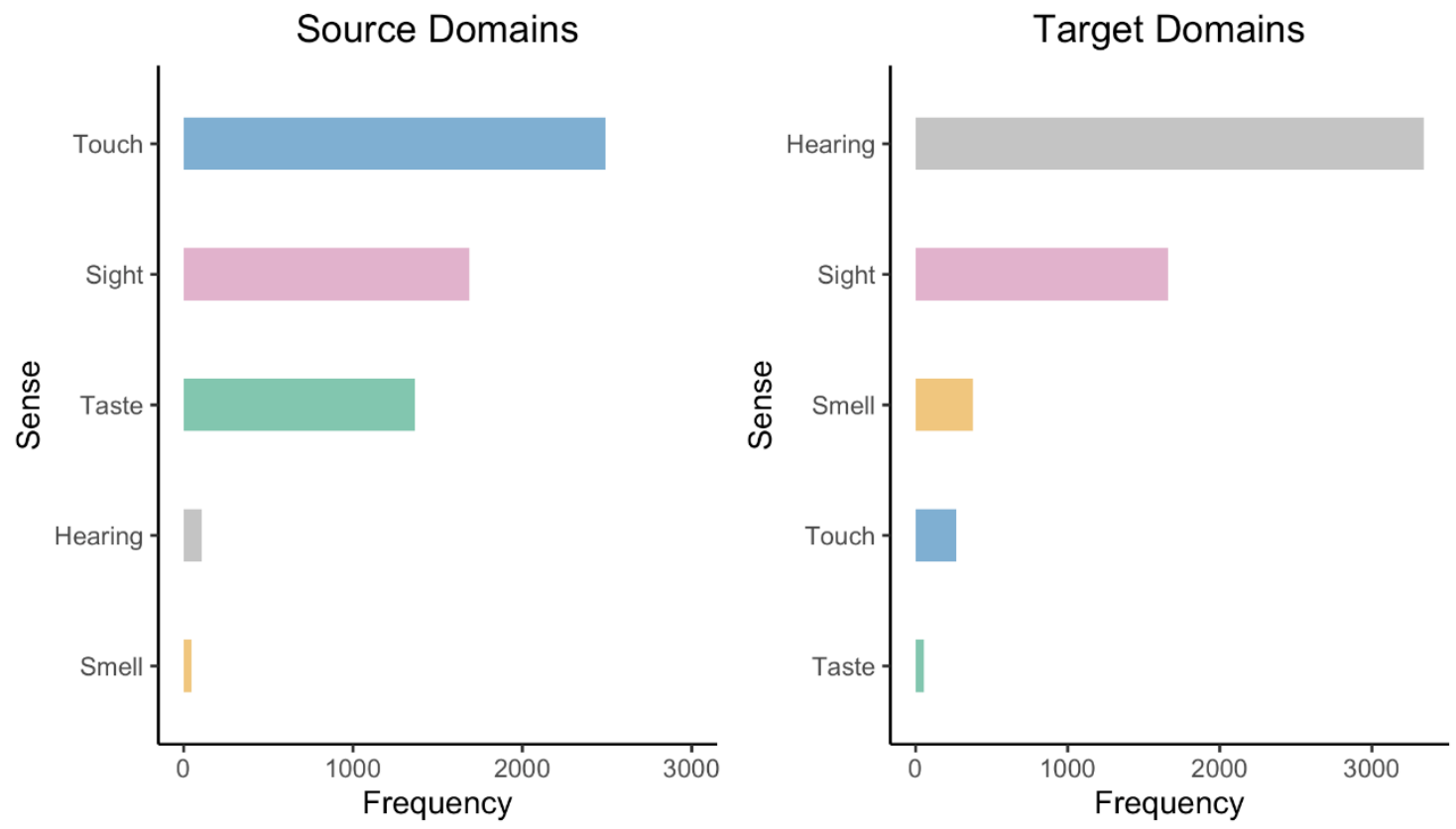

\section{Backward Instances}

There were synaesthetic occurrences in the backward direction as can be seen from Table 2 .

More precisely, transfers from taste to touch, from smell to taste and touch, from hearing to taste and touch and from sight to hearing, smell, taste and touch can be registered as "uncanonical" according to the directionality principle. Representative examples of the backward transfers are as follows:

(1) From taste to touch

Bunları okurken zannediyorum içinizi tatı bir sıcaklık saracak.

As you read them, I guess, a sweet warmness will embrace you.

(2) From smell to taste

...yemeğe hoş kokulu bir lezzet verir.

...it gives a pleasant-smelling taste to the meal.

(3) From smell to touch

Devrelerim senin kolonya kokulu dokunuşlarına hasret...

My circuits are longing for your cologne-scented touches... 
(4) From hearing to taste

...melodik bir tat vermekte...

...it gives a melodic taste...

(5) From hearing to touch

Bendeki geniş, mızmız, bıktırıcı bir ağrı.

Mine is a wide-spread, whiny, tiresome pain.

(6) From sight to hearing

Yanındaki kızın berrak sesini duydu birden.

He suddenly heard the clear voice of the girl next to him.

(7) From sight to taste

Çok tatlıymış... Yeşil tadı görünüyormuş üstlerinde.

They were very sweet... Their green taste appeared on them.

(8) From sight to touch

Çukurova'ya gitmişler ama "sarı sıcağın işkencesi", kara sıtma...

They went to Çukurova but the "torture of the yellow heat", black malaria...

\section{Transfer Types}

Opposing transfer directions (e.g., from sight to hearing and from hearing to sight) were compared with chi-square tests to classify the mapping tendencies in Turkish (see Zhao et al., 2018). Synaesthetic instances were identified by searching concordance lines which were accessed with perceptual adjectives as described in Methods above. As a result, the number of concordance lines as per source domain varied according to the number of perceptual adjectives. For example, 96,815 concordance lines with sight as the source were accessed using 135 sight-related adjectives and among them, 1496 instances from sight to hearing were identified. On the other hand, 10,267 concordance lines with hearing as the source were accessed using 31 hearing-related adjectives and 98 instances 
from hearing to sight were identified. To be able to compare frequencies of synaesthetic instances with different perceptual domains, raw frequencies were normalised per million using the computation below where $f$ is the raw frequency, $C$ is the number of concordance lines and $n f$ is the normalised frequency:

$$
n f=\frac{f\left(10^{6}\right)}{C}
$$

Chi-square tests were conducted based on normalised frequencies and the results were presented in Table 3.

\section{Table 3}

Normalised Frequencies (nf) of Synaesthetic Transfers per Million and the Differences $\left(x^{2}\right)$ between the Instances of Opposing Directions

\begin{tabular}{llcc}
\hline Source & Target & $n f$ & $x^{2}$ \\
\hline Sight & Hearing & 155 & $497^{*}$ \\
Hearing & Sight & 9545 & $6413^{*}$ \\
\hline Touch & Hearing & 31014 & \\
Hearing & Touch & 487 & \\
\hline Touch & Sight & 28040 & $3174^{*}$ \\
Sight & Touch & 1560 & \\
\hline Taste & Hearing & 60381 & $110641^{*}$ \\
Hearing & Taste & 390 & \\
\hline Taste & Sight & 45355 & $22125^{*}$ \\
Sight & Taste & 72 & \\
\hline Touch & Smell & 5948 & \\
& & & \\
\hline
\end{tabular}




\begin{tabular}{llcr} 
Smell & Touch & 9534 & \\
\hline Taste & Smell & 10812 & \\
Smell & Taste & $565.4^{*}$ \\
\hline Taste & Touch & 8613 & $15728^{*}$ \\
Touch & Taste & 1168 & \\
\hline Sight & Smell & $362728^{*}$ \\
Smell & Sight & 5609 & \\
\hline Smell & Hearing & 9534 & \\
Hearing & Smell & 0 & \\
& & & \\
\hline
\end{tabular}

Note. ${ }^{*} p<.0001$. Dominant directions are in bold.

Chi-square tests showed that there are significant differences between the frequency of all transfers types except for the transfers between smell and hearing. Transfers from smell to hearing can be identified as unidirectional because there were no instances with hearing as the source domain and smell as the target domain. In this regard, synaesthetic instances with smell and hearing can be said to comply with the directionality principle in the strictest sense.

Results indicated that all other transfer types in Turkish fall into the frequency-based pattern as there was a dominant direction which was significantly more frequent than the non-dominant direction (see Figure 5). Association types from hearing to sight, from touch to hearing, from touch to sight, from taste to hearing, from taste to sight, from taste to smell and from smell to sight are in accordance with the canonical hierarchy between senses. However, transfers from smell to touch and from taste to touch do not satisfy the rigid pattern of the directionality although they were more frequent than the 
conventional transfers (i.e., from touch to smell and from touch to taste). Results showed that there were no bidirectional transfer types in Turkish where mappings in both directions occur equally likely.

\section{Figure 5}

Transfer Directionality of Turkish Synaesthesia

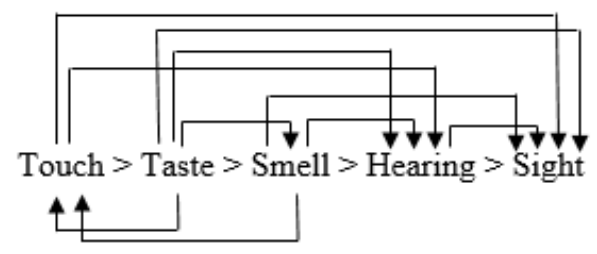

Note. Arrows to the right indicate conventional transfers and arrows to the left indicate unconventional transfers as per the directionality principle.

\section{Discussion}

The current study evaluated linguistic synaesthesia in Turkish. Our research questions, in particular, concerned the presence and the type of transfer patterns in comparison to other languages. No previous research to our knowledge has shown directionality in Turkish linguistic synaesthesia. We screened a large corpus with 50 million word tokens and extracted 5699 synaesthetic instances, which is comparable to Zhao et al. (2019) and considerably larger than the previous studies (e.g., Jo, 2019; Shen \& Cohen, 1998; Shen \& Gil, 2008; Strik Lievers, 2015; Ullmann, 1957). Such a large sample allowed for a comprehensive and a rigorous examination of linguistic synaesthesia in Turkish that is not limited to a genre, domain, register or period and thus, to test the universality claim of the directionality principle. Further, results were analysed not only with descriptive means but also with significance tests based on chi-squared statistics.

Do synaesthetic instances show a specific transfer pattern in Turkish? Findings clearly indicated that Turkish linguistic synaesthesia does follow a specific pattern rather than a random mapping between five senses. There could be ten possible transfer types involving two senses in the opposing 
directions (e.g., from sight to smell and from smell to sight) in a system of five senses. Results showed that nine transfer types in Turkish out of ten fit into the frequency-based pattern in which there was a significantly more frequent and thus, dominant direction. These transfers cannot be classified as unidirectional or bidirectional because there were backward transfers as well and significant differences between the frequencies of opposing associations. The only exception was the transfer from smell to hearing, which was unidirectional (i.e., rule-based) because the inverse transfer (i.e., from hearing to smell) was not available in our sample. Descriptive analyses showed that touch served as the most frequent source and hearing as the most frequent target. There were significant differences in frequencies between senses as the source and target domains except for sight, which was equally frequent in the source and target domain.

Results, overall, suggest that Turkish linguistic synaesthesia does not support the universality claim (Williams, 1976) of the directionality principle (Ullmann, 1957), which submits that cross-modal transfers in linguistic synaesthesia follow the hierarchy between senses (i.e., from lower to higher senses) with only unidirectional transfers. Generally speaking, findings in the present work are in line with the recent studies investigating synaesthesia in English (Strik Lievers, 2015), Italian (Strik Lievers, 2015), Mandarin Chinese (Zhao et al., 2019) and Korean (Jo, 2019), which argue that the directionality principle cannot be interpreted as a strictly rule-based constraint. On the other hand, predominant source (i.e., touch) and predominant target domains (i.e., hearing) in the current study not only comply with more recent studies but also support data from Ullmann (1957). In addition to the similarities summarised above, there are notable differences between Turkish and other languages from which recent and comparable data is available.

First, Turkish linguistic synaesthesia in the current study was more diverse than synaesthesia in the abovementioned languages. Out of 20 possible transfer types, 19 transfer types were found in Turkish compared to 17 transfer types in Korean, 15 transfer types in Mandarin Chinese, 16 transfer 
types in English and 14 transfer types in Italian. Instances from hearing to smell were not available in Mandarin Chinese, English or Italian either. ${ }^{6}$ However, the instances that were not found in Mandarin Chinese (i.e., from smell to touch, from hearing to touch, from hearing to taste and from smell to hearing), English (i.e., from smell to taste) and Italian (i.e., from hearing to touch, from taste to touch and from sight to smell) were extracted from the TNC in the present study. Second, there were no bidirectional transfers in Turkish as opposed to Mandarin Chinese. The bidirectional transfer between touch and taste in Mandarin Chinese was frequency-based in Turkish, in which the mapping from taste to touch was dominant over the mapping from touch to taste. Third, Turkish linguistic synaesthesia revealed two dominant transfers which proceed contrary to the cross-modal hierarchy. Normalised frequencies that were controlled for the number of concordance lines and thus, the number of perceptual adjectives demonstrated that synaesthetic occurrences from taste to touch and from smell to touch were significantly more frequent than their conventional counterparts (i.e., from touch to taste and from touch to smell). These mappings were either less frequent than the congruent ones or not available at all in English, Italian, Mandarin Chinese and Korean. Fourth, sight occurred more frequently than taste as a source domain (see Table 4) and touch occurred more frequently than taste as a target domain in Turkish linguistic synaesthesia (see Table 5). That said, all other source and target modes comply with the frequency order in other languages.

\section{Table 4}

Source Sensory Domains in Decreasing Order of Frequency (Percentage) In Turkish (Except for Taste and Sight), English, Italian and Korean

\footnotetext{
${ }^{6}$ Yet there was one instance from hearing to smell in Korean (Jo, 2019).
} 


\begin{tabular}{lrcccr}
\hline & Touch & Taste & Sight & Hearing & Smell \\
\hline Turkish & 43.72 & $\mathbf{2 3 . 9 7}$ & $\mathbf{2 9 . 6 3}$ & 1.88 & 0.80 \\
English & 49.3 & 25.7 & 21.8 & 3.0 & 0.2 \\
Italian & 55.6 & 20.2 & 19.1 & 4.6 & 0.2 \\
Korean & 37 & 33 & 20 & 7 & 3 \\
\hline
\end{tabular}

\section{Table 5}

Target Sensory Domains in Decreasing Order of Frequency (Percentage) In Turkish (Except for Taste and Touch), English, Italian and Korean

\begin{tabular}{lrcccr}
\hline & Hearing & Sight & Smell & Taste & Touch \\
\hline Turkish & 58.60 & 29.12 & 6.61 & $\mathbf{0 . 9 8}$ & $\mathbf{4 . 6 9}$ \\
English & 52.3 & 28.0 & 12.4 & 5.3 & 2.1 \\
Italian & 50.2 & 42.5 & 3.8 & 3.0 & 0.2 \\
Korean & 50 & 26 & 16 & 5 & 3 \\
\hline
\end{tabular}

How to account for the commonality and variation in linguistic synaesthesia between Turkish and other languages? It is worth discussing this question as possible answers are crucial not only for the universality claim but also for the very nature of the linguistic synaesthesia beyond a given language. Universalist accounts versus cross-linguistic and cross-cultural variation have been an ongoing debate in the metaphor literature for a long time (see O’Meara et al., 2019). Grounded-embodied views predict that there should be some kind of commonality across languages in how metaphors are formed and how one category is mapped onto another as long as we have similar bodies and thus, similar sensorimotor systems (Lakoff \& Johnson, 1999). Proponents of this view claim that embodiment has a neurological basis (see Cohen \& Leung, 2009 for "pre-wired embodiments"), which then entails a 
universal and rigid transfer hierarchy in cross-modal associations of linguistic synaesthesia (Marks, 1996; Rakova, 2003; Ronga et al., 2012). For example, results in the current study show that Turkish users mostly speak of their auditory and visual experiences as in the case of soğuk ses - cold voice and sert bakmak - to look hard (to scowl) because hearing and sight were the most frequent target domains. This is a pattern that seems to be fairly consistent across languages. The dominance of hearing and sight as a target over other sensory domains can be explained with the fact that humans already display a robust tendency to rely more on visual information than other forms of sensory information (Colavita, 1974) as reported in several experiments (e.g., Ngo et al., 2010; Sinnett et al., 2007). ${ }^{7}$ Similarly, the current findings along with the previous ones demonstrate that individuals, to a large extent, refer to the tactile perception to describe their experience in other sensory modalities. Tact, as a proximal sense, is assumed to be more "accessible" compared to the other sensory modalities due to the direct contact between the subject and the object of perception (Paradis \& Eeg-Olofsson, 2013; Shen \& Aisenman, 2008). Further, tactile sensation is broad enough to define our relation with the physical environment and very experience of existence in time and space (O'Shaughnessy, 1989). The essential position of touch might account for its predominance as the source of transfers regardless of language or culture. On the other hand, growing evidence has shown that there is also much room for linguistic and cultural variation in perceptual language (Kövecses, 2005; Majid \& Levinson, 2011), which was reflected in the present study as described above. For example, although taste is regarded as a "lower sense" and thus, more accessible than sight, Turkish users refer to visual perception more frequently than gustatory perception to describe perceptual experiences compared to users of other languages. This might be related to the versatility of visual lexemes in Turkish, in particular, lexemes that describe colours (e.g.,

\footnotetext{
${ }^{7}$ However, why hearing but not sight is the most frequent target despite the fact that vision is dominant over audition (Witten \& Knudsen, 2005) requires further attention (see Strik Lievers, 2015).
} 
Bayraktar, 2004). Given the available evidence and our findings, we conclude that both biological propensities and linguistic/cultural variables play a role in the formation of synaesthetic occurrences. Future research can explore "to what extent" biological and linguistic/cultural factors are involved in linguistic synaesthesia.

It is tempting to speculate about linguistic universals based on the available cross-linguistic evidence of synaesthesia. However, it is equally important to consider the terminological and methodological differences between the abovementioned studies such as the very definition of synaesthesia (see Winter, 2019b), perceptual lexemes identified to extract instances, perceptual taxonomy, corpus size and type, size of the synaesthetic sample, and data analysis. Most importantly, perceptual taxonomy requires further clarification across studies. As Winter (2019a) noted, regardless of the taxonomy system, our perceptual classification is arbitrary and thus, far from reflecting extralinguistic experiences in the "real world". Instances between taste and smell, in particular, are to be interpreted with caution as taste and smell work together within the chemosensory perception (Hummel \& Welge-Lüssen, 2006) and in turn, have reflections in the perceptual language (e.g., Croijmans \& Majid, 2016). In the present study, instances that "describe" the smell of edibles were excluded from the sample (e.g., kokulu şeker-scented candy or kokulu baharatlar-scented spices) although the described nouns were in the list of perceptual lexemes. Only one instance from smell to taste (i.e., kokulu lezzet - scented taste) was then left in the sample because the olfaction-related adjective modifies the very experience of tasting rather than a certain type of food in this occurrence. That said, all "synaesthetic" instances between smell and taste are up to debate in the framework of the discussion above and this might account for the differences between languages.

Under the broader umbrella of embodied cognition and sensory linguistics, linguistic synaesthesia represents a promising case as it speaks to the network between the sensorimotor system, language and culture. Growing corpus data from a wide range of languages give us a varied picture of 
linguistic synaesthesia used in remarkably variable contexts. However, the number of languages in which synaesthesia has been investigated is still limited. Studies on the uncharted languages and uncharted perceptual domains are much required to shed further light on the universality claim of the directionality principle and thus, the mechanism behind this colourful phenomenon. 


\section{References}

Aksan, Y., Aksan, M., Koltuksuz, A., Sezer, T., Mersinli, Ü., Demirhan, U. U., Yilmazer, H., Kurtoglu, Ö., Atasoy, G., Öz, S., \& Yildiz, I. (2012). Construction of the Turkish national corpus (TNC). In N. Calzolari, K. Choukri, T. Declerck, M. U. Doğan, B. Maegaard, J. Mariani, A. Moreno, J. Odijk, \& S. Piperidis (Eds.), Proceedings of the $8^{\text {th }}$ International Conference on Language Resources and Evaluation (LREC 2012) (pp. 3223-3227). European Language Resources Association (ELRA).

Baroni, M., Bernardini, S., Ferraresi, A., \& Zanchetta, E. (2009). The waCky wide web: A collection of very large linguistically processed web-crawled corpora. Language Resources and Evaluation, 43(3), 209-226. https://doi.org/10.1007/s10579-009-9081-4

Bayraktar, N. (2004). Conceptual and semantic properties of colour names kara and siyah in Turkish. Dil Dergisi, 126, 56-76.

Chen, K.-J., Huang, C.-R., Chang, L.-P., \& Hsu, H.-L. (1996). Sinica corpus: Design methodology for balanced corpora. In B.-S. Park \& J.-B. Kim (Eds.), Proceedings of the $11^{\text {th }}$ Pacific Asia Conference on Language, Information and Computation (PACLIC 11) (pp. 167-176). Kyung Hee University.

Cohen, D., \& Leung, A. K. Y. (2009). The hard embodiment of culture. European Journal of Social Psychology, 39(7), 1278-1289. https://doi.org/10.1002/ejsp.671

Colavita, F. B. (1974). Human sensory dominance. Perception \& Psychophysics, 16(2), 409-412. https://doi.org/10.3758/BF03203962

Craig, A. D. (2002). How do you feel? Interoception: the sense of the physiological condition of the body. Nature Reviews Neuroscience, 3(8), 655-666. https://doi.org/10.1038/nrn894

Croijmans, I., \& Majid, A. (2016). Not all flavor expertise is equal: The language of wine and coffee experts. PLOS ONE, 11(6), e0155845. https://doi.org/10.1371/journal.pone.0155845

Day, S. A. (1996). Synaesthesia and synaesthetic metaphors. Psyche, 2(32), 2-32.

Erzsébet, P. D. (1974). Synaesthesia and poetry. Poetics, 3(3), 23-44. https://doi.org/https://doi.org/10.1016/0304-422X(74)90021-7

Evans, N., \& Wilkins, D. (2000). In the mind's ear: the semantic extensions of perception verbs in australian languages. Language, 76(3), 546-592. https://doi.org/10.2307/417135

Gibbs, R. (2010). The wonderful, chaotic, creative, heroic, challenging world of researching and applying 
metaphor. In G. Low, Z. Todd, A. Deignan, \& L. Cameron (Eds.), Researching and applying metaphor in the real world (pp. 1-18). John Benjamins Publishing Company. https://doi.org/10.1075/hcp.26.01gib

Gray, R. (2005). On the concept of a sense. Synthese, 147(3), 461-475. https://doi.org/10.1007/s11229005-1334-1

Hummel, T., \& Welge-Lüssen, A. (2006). Taste and smell: An update. Karger.

Hunston, S. (2002). Corpora in applied linguistics. Ernst Klett Sprachen.

Jo, C. (2019). A corpus-based analysis of synesthetic metaphors in Korean. Linguistic Research, 36(3), 459-483. https://doi.org/10.17250/khisli.36.3.201912.005

Kang, B., \& Kim, H. (2004). Sejong Korean Corpora in the Making. In M. T. Lino, M. F. Xavier, F. Ferreira, R. Costa, \& R. Silva (Eds.), Proceedings of the $4^{\text {th }}$ International Conference on Language Resources and Evaluation (LREC 2004) (pp. 1747-1750). European Language Resources Association (ELRA).

Keeley, B. L. (2002). Making sense of the senses: Individuating modalities in humans and other animals. Journal of Philosophy, 99(1), 5-26. https://doi.org/10.2307/3655759

Kövecses, Z. (2005). Cross-cultural variation. In Metaphor in culture: Universality and variation (pp. 6787). Cambridge University Press. https://doi.org/10.1017/CBO9780511614408.006

Lakoff, G., \& Johnson, M. (1980a). Metaphors we live by. University of Chicago Press.

Lakoff, G., \& Johnson, M. (1980b). The metaphorical structure of the human conceptual system. Cognitive Science, 4(2), 195-208. https://doi.org/10.1016/S0364-0213(80)80017-6

Lakoff, G., \& Johnson, M. (1999). Philosophy in the flesh: The embodied mind and its challenge to Western thought. Basic Books.

Lynott, D., \& Connell, L. (2009). Modality exclusivity norms for 423 object properties. Behavior Research Methods, 41(2), 558-564. https://doi.org/10.3758/BRM.41.2.558

Macpherson, F. (2011). Taxonomising the senses. Philosophical Studies, 153(1), 123-142. https://doi.org/10.1007/s11098-010-9643-8

Majid, A., \& Levinson, S. C. (2011). The senses in language and culture. The Senses and Society, 6(1), 518. https://doi.org/10.2752/174589311X12893982233551 
Majid, A., Roberts, S. G., Cilissen, L., Emmorey, K., Nicodemus, B., O’Grady, L., Woll, B., LeLan, B., De Sousa, H., Cansler, B. L., Shayan, S., De Vos, C., Senft, G., Enfield, N. J., Razak, R. A., Fedden, S., Tufvesson, S., Dingemanse, M., Ozturk, O., ... Levinson, S. C. (2018). Differential coding of perception in the world's languages. Proceedings of the National Academy of Sciences of the United States of America, 115(45), 11369-11376. https://doi.org/10.1073/pnas.1720419115

Marks, L. E. (1996). On perceptual metaphors. Metaphor and Symbolic Activity, 11(1), 39-66. https://doi.org/10.1207/s15327868ms1101 3

Merleau-Ponty, M. (1960). Phenomenology of perception. Routledge.

Ngo, M. K., Sinnett, S., Soto-Faraco, S., \& Spence, C. (2010). Repetition blindness and the Colavita effect. Neuroscience Letters, 480(3), 186-190. https://doi.org/10.1016/i.neulet.2010.06.028

O'Meara, C., Speed, L. J., San Roque, L., \& Majid, A. (2019). Perception metaphors: A view from diversity. In L. J. Speed, C. O’Meara, L. San Roque, \& A. Majid (Eds.), Perception metaphors (pp. 1-16). John Benjamins Publishing Company. https://doi.org/10.1075/celcr.19.01ome

O'Shaughnessy, B. (1989). The sense of touch. Australasian Journal of Philosophy, 67(1), 37-58. https://doi.org/10.1080/00048408912343671

R Core Team. (2020). R: A language and environment for statistical computing. R Foundation for Statistical Computing. https://www.r-project.org/.

Rakova, M. (2003). The extent of the literal: Metaphor, polysemy and theories of concepts. Springer.

Ramachandran, V. S., \& Hubbard, E. M. (2001). Synaesthesia--a window into perception, thought and language. Journal of Consciousness Studies, 8(12), 3-34.

Ronga, I., Bazzanella, C., Rossi, F., \& Iannetti, G. (2012). Linguistic synaesthesia, perceptual synaesthesia, and the interaction between multiple sensory modalities. Pragmatics \& Cognition, 20(1), 135167. https://doi.org/10.1075/pc.20.1.06ron

San Roque, L., Kendrick, K. H., Norcliffe, E., Brown, P., Defina, R., Dingemanse, M., Dirksmeyer, T., Enfield, N. J., Floyd, S., Hammond, J., Rossi, G., Tufvesson, S., Van Putten, S., \& Majid, A. (2015). Vision verbs dominate in conversation across cultures, but the ranking of non-visual verbs varies. Cognitive Linguistics, 26(1), 31-60. https://doi.org/10.1515/cog-2014-0089

Shen, Y. (1997). Cognitive constraints on poetic figures. Cognitive Linguistics, 8(1), 33-72. https://doi.org/10.1515/cogl.1997.8.1.33 
Shen, Y., \& Aisenman, R. (2008). "Heard melodies are sweet, but those unheard are sweeter": Synaesthetic metaphors and cognition. Language and Literature, 17(2), 107-121. https://doi.org/10.1177/0963947007088222

Shen, Y., \& Cohen, M. (1998). How come silence is sweet but sweetness is not silent: A cognitive account of directionality in poetic synaesthesia. Language and Literature, 7(2), 123-140. https://doi.org/10.1177/096394709800700202

Shen, Y., \& Gil, D. (2008). Sweet fragrances from Indonesia: a universal principle governing directionality in synaesthetic metaphors. In J. Auracher \& W. van Peer (Eds.), New beginnings in literary studies (pp. 49-70). Cambridge Scholars Publishing.

Sinnett, S., Spence, C., \& Soto-Faraco, S. (2007). Visual dominance and attention: The Colavita effect revisited. Perception \& Psychophysics, 69(5), 673-686. https://doi.org/10.3758/BF03193770

Strik Lievers, F. (2015). Synaesthesia: A corpus-based study of cross-modal directionality. Functions of Language, 22(1), 69-95. https://doi.org/10.1075/fol.22.1.04str

Strik Lievers, F., \& Felice, I. (2019). Metaphors and perception in the lexicon: A diachronic perspective. In Perception Metaphors (pp. 85-104). John Benjamins Publishing Company.

\section{https://doi.org/10.1075/celcr.19.05str}

Strik Lievers, F., Xu, H., \& Xu, G. (2013). A methodology of the extraction of lexicalized synaesthesia from corpora [Conference presentation]. ICLI9 (International Congress of Linguists), Geneva, Switzerland.

TNC Team. (2018). Turkish National Corpus (No. 3). Mersin University. https://v3.tnc.org.tr.

Tognini-Bonelli, E. (2001). Corpus linguistics at work. John Benjamins Publishing Company.

Tsur, R. (1992). Toward a theory of cognitive poetics. North-Holland Publishing Company.

Ullmann, S. (1957). The principles of semantics ( $2^{\text {nd }}$ ed.). Blackwell.

Viberg, Å. (1983). The verbs of perception: A typological study. Linguistics, 21(1), 123-162. https://doi.org/10.1515/ling.1983.21.1.123

Ward, J., \& Simner, J. (2003). Lexical-gustatory synaesthesia: Linguistic and conceptual factors. Cognition, 89(3), 237-261. https://doi.org/10.1016/S0010-0277(03)00122-7

Werning, M., Fleischhauer, J., \& Beşeoğlu, H. (2001). The cognitive accesibility of synaesthetic 
metaphors. In R. Sun \& N. Miyake (Eds.), Proceedings of the 28th Annual Conference of the Cognitive Science Society (pp. 2365-2370). Cognitive Science Society.

Williams, J. M. (1976). Synaesthetic adjectives: A possible law of semantic change. Language, 52(2), 461. https://doi.org/10.2307/412571

Winter, B. (2019a). Synaesthetic metaphors are neither synaesthetic nor metaphorical. In L. J. Speed, C. O’Meara, L. S. Roque, \& A. Majid (Eds.), Perception metaphors (pp. 105-126). John Benjamins Publishing Company. https://doi.org/10.1075/celcr.19.06win

Winter, B. (2019b). Synesthesia and metaphor. In Sensory linguistics: Language, perception and metaphor (pp. 67-77). John Benjamins Publishing Company. https://doi.org/10.1075/celcr.20

Wise, H. (1997). The vocabulary of modern French. Routledge.

Witten, I. B., \& Knudsen, E. I. (2005). Why seeing is believing: merging auditory and visual worlds. Neuron, 48(3), 489-496. https://doi.org/10.1016/j.neuron.2005.10.020

Yu, N. (1992). A possible semantic law in synaesthetic transfer. SECOL Review, 16(1), 20-40.

Yu, N. (2003). Synesthetic metaphor: A cognitive perspective. Journal of Literary Semantics, 32(1), 1934. https://doi.org/10.1515/ilse.2003.001

Zhao, Q., Huang, C. R., \& Ahrens, K. (2019). Directionality of linguistic synesthesia in Mandarin: A corpusbased study. Lingua, 232, 102744. https://doi.org/10.1016/j.lingua.2019.102744

Zhao, Q., Huang, C. R., \& Long, Y. (2018). Synaesthesia in Chinese: A corpus-based study on gustatory adjectives in Mandarin. Linguistics, 56(5), 1167-1194. https://doi.org/10.1515/ling-2018-0019 